\title{
Association of laryngeal cancer with vocal cord leukoplakia and associated risk factors in 1,184 patients diagnosed in otorhinolaryngology practices in Germany
}

\author{
KAREL KOSTEV $^{1}$, LOUIS E.C. JACOB ${ }^{2}$, MATTHIAS KALDER ${ }^{3}$, \\ ANDREAS M. SESTERHENN ${ }^{4}$ and DAVID ULRICH SEIDEL ${ }^{4}$
}

\begin{abstract}
${ }^{1}$ Department of Epidemiology, Quintiles IMS, D-60598 Frankfurt, Germany; ${ }^{2}$ Faculty of Medicine, University of Paris V, 75006 Paris, France; ${ }^{3}$ Department of Gynecology and Obstetrics, Philipps University of Marburg, D-35037 Marburg; ${ }^{4}$ Department of Otorhinolaryngology, Head and Neck Surgery, Solingen Municipal Hospital, D-42653 Solingen, Germany
\end{abstract}

Received February 24, 2018; Accepted March 20, 2018

DOI: $10.3892 / \mathrm{mco} .2018 .1592$

\begin{abstract}
The aim of the present study was to analyze the association between laryngeal cancer and vocal cord leukoplakia in a large nationwide practice database. The study sample included 1,184 patients aged 18-90 years from 113 otorhinolaryngology practices in Germany who were diagnosed with vocal cord leukoplakia between 2007 and 2014. The rates of laryngeal cancer diagnosis within 5 years and the associated risk factors were analyzed. Within 6 months of initial diagnosis of vocal cord leukoplakia, $11 \%$ of the patients were diagnosed with laryngeal cancer. Between 7 months and 5 years after the diagnosis of leukoplakia, laryngeal cancer was diagnosed in $7.6 \%$ of the patients. Overall, within 5 years of leukoplakia diagnosis, $18.6 \%$ of the patients were diagnosed with laryngeal cancer (26.1\% of men and $6.3 \%$ of women; $\mathrm{P}<0.001$ ). Moreover, $31.3 \%$ of patients aged $>65$ years, $16.1 \%$ of patients aged $50-65$ years and $7.6 \%$ of patients aged $<50$ years were diagnosed with laryngeal cancer $(\mathrm{P}<0.001)$. Patients aged $>65$ and those aged $50-65$ years had a higher risk of being diagnosed with laryngeal cancer [odds ratio $(\mathrm{OR})=4.90$ and 2.55 , respectively]. Furthermore, the risk of being diagnosed with laryngeal cancer was higher in men compared with that in women $(\mathrm{OR}=4.09)$. In the present real-world analysis, the rate of laryngeal cancer underlying vocal cord leukoplakia at first diagnosis appeared to be higher compared with the secondary malignant transformation rate occurring later during the course of the disease. Risk factors for laryngeal cancer included advanced age and male sex.
\end{abstract}

Correspondence to: Professor Karel Kostev, Department of Epidemiology, Quintiles IMS, 108 Darmstädter Landstraße, D-60598 Frankfurt, Germany

E-mail:kkostev@de.imshealth.com

Key words: laryngeal cancer, larynx, leukoplakia, incidence, risk factors, otorhinolaryngology practices, nationwide practice database

\section{Introduction}

The clinical and descriptive term 'leukoplakia' denotes a white patch on a mucosal surface identified on macroscopic examination. The underlying pathology includes various lesions, such as squamous hyperplasia with or without keratosis, epithelial dysplasia of various grades of severity, carcinoma in situ (CIS) or invasive carcinoma. Therefore, biopsy and histological examination are warranted to obtain a definitive diagnosis $(1,2)$. The most important etiological factors for the development of laryngeal precancerous lesions, as well as malignant transformation to invasive carcinoma, are tobacco and alcohol abuse. In recent years, gastroesophageal reflux has also been considered to play a causative role (3). Other possible etiological factors are occupational hazards (e.g., asbestos), nutritional deficiencies, vocal abuse, chronic infections and hormonal disorders $(4,5)$.

The association of vocal cord leukoplakia with laryngeal carcinoma has been the subject of numerous clinical studies and meta-analyses over the past decades, which have been mainly based on data from hospitals $(3,6,7)$. The rates of carcinoma underlying leukoplakia at first diagnosis and the secondary malignant transformation rates in the later course of the disease vary widely between studies, which is due to a marked sampling or case selection bias of hospital-based studies when compared with the general population, selecting more severe cases and higher transformation rates in specialized centers (6). The only population-based study on vocal cord leukoplakia and laryngeal cancer with supposedly less biased data included a limited number of cases, only 108 over a period of 49 years (8). Current data from the German healthcare system on vocal cord leukoplakia and its association with laryngeal cancer have yet to be published.

The status of otorhinolaryngology practices in particular, with their role as first diagnostic gatekeepers, has yet to be examined. Thus, the aim of the present study was to investigate the current epidemiological status of vocal cord leukoplakia and its association with laryngeal cancer in otorhinolaryngology practices in Germany, based on data from a large and representative nationwide practice database. 


\section{Materials and methods}

Database. The Disease Analyzer database (QuintilesIMS, Frankfurt, Germany) compiles drug prescriptions, diagnoses, and basic medical and demographic data obtained directly and in anonymous format from computer systems used in physicians' practices (9). Diagnoses (ICD-10), prescriptions [Anatomical Therapeutic Chemical (ATC) Classification System], and the quality of reported data are monitored by QuintilesIMS based on a number of criteria, such as completeness of documentation and association between diagnoses and prescriptions, among others.

In Germany, the sampling methods used for the selection of physicians' practices were considered appropriate for obtaining a representative database of such practices (9). The sampling method for the Disease Analyzer database is based on summary statistics from all physicians in Germany published yearly by the German Medical Association. IMS uses these statistics to determine the panel design according to specialist group, German federal state, community size category, and age of the physician. This panel design forms the basis for the selection of practices included in the Disease Analyzer. The sampling plan is subdivided into 8 geographical regions, which are groupings of the 16 German federal states. This stratification results in 176 cells, derived from the summary statistics for the specialist fields, and is proportional to the summary statistics for the German federal states. Within each region and specialist field, a minimum of 7 physicians must be sampled to allow for estimates for each region at the specialist field level. The database has already been used in several studies focusing on cancer (10-12).

Study population. The study sample included patients aged 18-90 years, from 113 otorhinolaryngology practices, who were diagnosed with vocal cord leukoplakia (ICD-10: J38.3, plus an original note by the physician containing the term 'leukoplakia') between January 2007 and December 2014 (index date). Patients were followed up for a maximum of 5 years. In order to guarantee the accuracy of the estimation of the progression of leukoplakia to laryngeal cancer, patients with a documented diagnosis of cancer (C00-C99) prior to the index date were excluded.

Study outcome and independent variables. The primary outcome measure was the rate of patients with a confirmed diagnosis of malignant neoplasm of the larynx, including malignant neoplasm of the glottis (C32.0), malignant neoplasm of overlapping sites of the larynx (C32.8) and unspecified malignant neoplasm of the larynx (C32.9). Malignant neoplasms of the supraglottis (C32.1), subglottis (C32.2) and laryngeal cartilage (C32.3) were excluded. Independent variables included age and sex.

Statistical analysis. Descriptive analyses were obtained for all variables and mean \pm standard deviation (SD) was calculated for normally distributed variables. Kaplan-Meier curves were used to analyze the percentage of patients who developed laryngeal cancer over time in the different sex and age groups. Finally, multivariate Cox regression models (dependent variable, incidence of cancer diagnosis) were used to determine laryngeal cancer risk when adjusting for age and sex. P-values $<0.05$ were considered to indicate statistically significant differences. Analyses were performed using SAS software, version 9.4 (SAS Institute, Inc., Cary, NC, USA).

\section{Results}

Patient characteristics. Socio-demographic data pertaining to the individuals included in the present study are shown in Table I. A total of 1,184 patients in 113 practices were available for analysis. The mean age was 58.2 years (SD, 14.0 years), and $61.8 \%$ of patients were men.

Laryngeal cancer diagnosis by age and sex. Kaplan-Meier curves for the diagnosis of laryngeal cancer are shown in Figs. 1 and 2. Within 6 months of initial diagnosis of vocal cord leukoplakia, $11 \%$ of the patients were diagnosed with laryngeal cancer. Between 7 months and 5 years after leukoplakia diagnosis, laryngeal cancer was diagnosed in $7.6 \%$ of the patients. Overall, within 5 years of leukoplakia diagnosis, $18.6 \%$ of the patients were diagnosed with laryngeal cancer (26.1\% of men and $6.3 \%$ of women; log-rank P-value $<0.001$, Fig. 1). Moreover, $31.3 \%$ of individuals aged $>65$ years, $16.1 \%$ of individuals aged 50-65 years, and 7.6\% of individuals aged $<50$ years were diagnosed with laryngeal cancer (logrank P-value $<0.001$, Fig. 2). Similar trends were observed for the three different age groups among men $(38.4,21.7$ and $14.7 \%$, respectively; log-rank P-value $<0.001)$ and women $(14.5$, 4.4 and $0.9 \%$, respectively; log-rank P-value $<0.003$ ).

Among patients who developed laryngeal cancer, $46.6 \%$ were diagnosed with malignant neoplasm of the glottis (ICD10: C32.0), $1.5 \%$ with malignant neoplasm of overlapping sites of the larynx (ICD-10: C32.8), and 51.9\% with unspecified malignant neoplasm of the larynx (C32.9). The results of the Cox regression model are presented in Table II. Patients aged $>65$ and those aged 50-65 years were at a higher risk of being diagnosed with laryngeal cancer $(\mathrm{OR}=4.90$ and 2.55 , respectively). Furthermore, the risk of developing laryngeal cancer was higher in men compared with that in women $(\mathrm{OR}=4.09)$.

\section{Discussion}

Using data from a large nationwide practice database, the present study analyzed the association of laryngeal cancer with vocal cord leukoplakia in 1,184 patients from 113 otorhinolaryngology practices in Germany. Within 6 months of initial diagnosis of vocal cord leukoplakia, $11.0 \%$ of the patients were diagnosed with laryngeal cancer, while between 7 months and 5 years after initial leukoplakia diagnosis, $7.6 \%$ of the patients were diagnosed with laryngeal cancer. Male sex and older age were found to be risk factors for the diagnosis of laryngeal cancer in these patients.

The association between laryngeal leukoplakia and cancer has been the subject of numerous clinical studies and metaanalyses in recent decades. The terminology and classification of this spectrum of lesions has changed markedly over time. While in early studies the term 'keratosis' was used as a clinical term interchangeably with leukoplakia (6), in current terminology 'keratosis' is strictly reserved for a histological 
Table I. Characteristics of patients with an initial diagnosis of vocal cord leukoplakia $(n=1,184)$ included in the present study (QuintilesIMS Disease Analyzer database).

\begin{tabular}{lr}
\hline Demographic characteristics & No. $(\%)$ \\
\hline Age (years), mean \pm standard deviation & $58.2(14.0)$ \\
$\quad<50$ & $346(29.2)$ \\
$<50-65$ & $462(39.0)$ \\
$>65$ & $376(31.8)$ \\
Sex & \\
Female & $452(38.2)$ \\
Male & $732(61.8)$ \\
\hline
\end{tabular}

Table II. Multivariate Cox regression model for the risk of laryngeal cancer diagnosis in patients with an initial diagnosis of vocal cord leukoplakia.

\begin{tabular}{lcc}
\hline Variables $^{\mathrm{a}}$ & OR $(95 \% \mathrm{CI})^{\mathrm{a}}$ & P-value $^{\mathrm{a}}$ \\
\hline $50-65$ vs. $<50$ years & $2.55(1.36-4.82)$ & 0.001 \\
$>65$ vs. $<50$ years & $4.90(2.66-9.03)$ & $<0.001$ \\
Male vs. female & $4.09(2.42-6.90)$ & $<0.001$ \\
\hline
\end{tabular}

aAdjusted for age and sex. OR, odds ratio; CI, confidence interval.

finding of a keratin layer on squamous epithelium (3). Moreover, the histological spectrum of laryngeal precancerous lesions underlying the clinical finding of vocal cord leukoplakia has been classified using a variety of grading systems over the years. Early classifications subdivided histology into keratosis without atypia (KWOA) vs. keratosis with atypia (KWA), or into grades I, II and III according to Kleinsasser (hyperplasia without atypia, hyperplasia with atypia and CIS, respectively) (13-17). Other histological grading systems, such as those for squamous intraepithelial neoplasia (SIN) or laryngeal intraepithelial neoplasia (LIN), were subsequently developed $(18,19)$. The most frequently used classifications today are the SIN system, the Ljubljana classification of squamous intraepithelial lesions (SIL) and, particularly, the WHO dysplasia system with a subdivision into hyperplasia, mild, moderate and severe dysplasia, and CIS (20-23).

In 1991, Bouquot and Gnepp published a review of studies on malignant transformation rates of various laryngeal precancerous lesions: Laryngeal keratosis (leukoplakia according to the authors' terminology), KWOA vs. KWA and laryngeal CIS (6). The studies revealed malignant transformation rates of $0.6-39.7 \%$ for laryngeal keratosis, $0.0-16 \%$ for KWOA, 5.6-40.0\% for KWA and 3.5-90.0\% for laryngeal CIS.

In 2008, Isenberg et al reviewed 15 clinical studies on vocal cord leukoplakia between 1960 and 2005 and included their own data from 136 patients (7). A total of 3,107 cases were included. Histological examination revealed no dysplasia in $53.6 \%$, mild to moderate dysplasia in $33.5 \%$ and CIS in $15.2 \%$ of the biopsies. The malignant transformation rates in

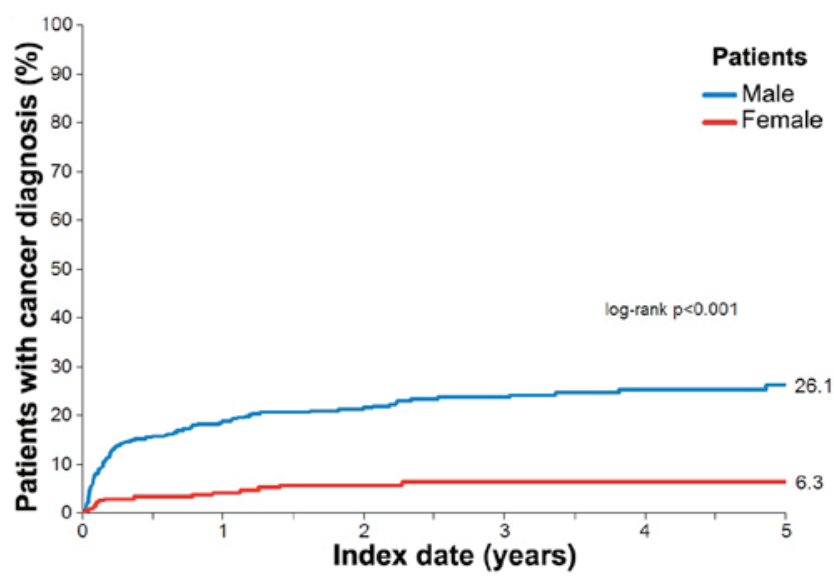

Figure 1. Kaplan-Meier curves for time to laryngeal cancer diagnosis in patients with vocal cord leukoplakia by sex.

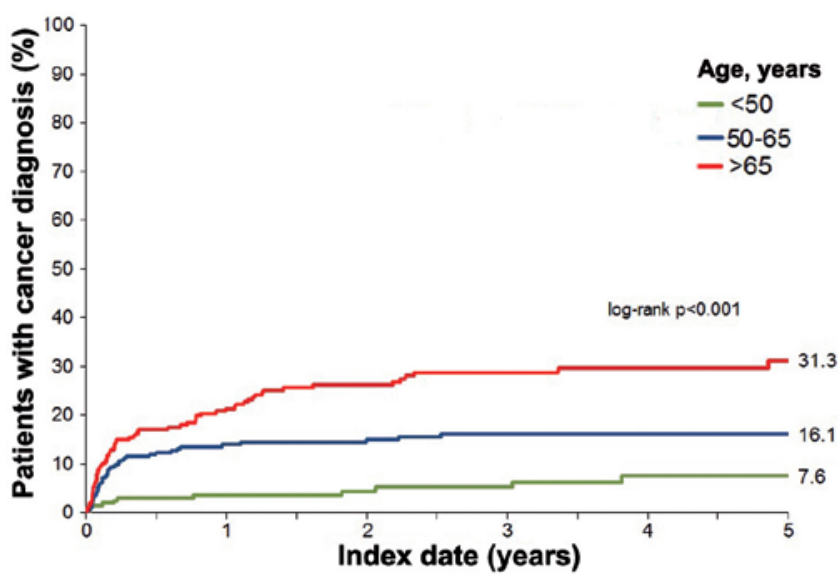

Figure 2. Kaplan-Meier curves for time to laryngeal cancer diagnosis in patients with vocal cord leukoplakia by age group.

lesions with no dysplasia, mild to moderate dysplasia and CIS were $3.7,10.1$ and $18.1 \%$, respectively. The overall malignant transformation rate (all lesions from all studies) was $8.2 \%$.

In 2009, Gale et al reviewed 9 clinical studies with a variety of different histological classifications between 1982 and 2003 $(n=2,841$ patients) and added their own data spanning 25 years ( $n=1,268$ patients) (4). Corresponding grades of different classification systems were pooled and the following malignant transformation rates were observed: Group of squamous hyperplasia and KWOA, 0-4.1\%; group of mild dysplasia, SIN I, LIN I and basal-parabasal hyperplasia, 0-11.5\%; group of moderate dysplasia, SIN II and LIN II, 4-24\%; and group of severe dysplasia, SIN III, LIN III, atypical hyperplasia and CIS, 9.3-57\%. The total transformation rates of all grades together ranged from $2.3 \%$ in the authors' own data to $21.4 \%$ in a study by Blackwell et al (24). The authors' own data represents the largest study on the topic to date, and reported transformation rates that were substantially lower compared with previous studies: $1.1 \%$ in squamous hyperplasia and basal/parabasal cell hyperplasia, $9.5 \%$ in atypical hyperplasia and $2.3 \%$ in all grades combined.

While the aforementioned clinical studies analyzed the malignant transformation rate of various precancerous lesions 
with known histology, the perspective of primary healthcare providers in daily otorhinolaryngology practice is based on a population with leukoplakia of initially unknown histology. As leukoplakia is merely a descriptive clinical term without any histological or prognostic implications, it is important to bear in mind that a certain percentage of these leukoplakias already contain carcinoma at the time of first presentation. Reliable and unbiased data on the rates of carcinoma underlying vocal cord leukoplakia at first presentation in contrast to secondary malignant transformation in the later course of the disease is scarce in the literature. In 1991, Bouquot et al published the first population-based study on laryngeal keratosis (leukoplakia according to the authors' terminology) and laryngeal cancer in the literature (8). Histological examination revealed underlying invasive cancer in $12 \%$ (carcinoma at diagnosis), while the secondary malignant transformation rate (carcinoma after diagnosis) was merely $1 \%$. In comparison to 10 previous studies with hospital-based data from 1953-1983, which reported carcinoma at diagnosis in 3.5-66.7\% and carcinoma after diagnosis in $0.0-42.0 \%$ of the patients, the rates of the present study were at the lower end of the spectrum, which may be explained by the minimal sampling/case selection bias in a population-based study when compared to hospital-based studies.

The results of the present German study of a representative nationwide practice database are in line with the findings of Bouquot's population-based study. The Kaplan-Meier curves revealed a steep increase in laryngeal cancer diagnoses within 6 months after the initial diagnosis of leukoplakia, followed by a flat increase towards the end of the study period. The initial steep increase most likely represents cases with carcinoma underlying the initial leukoplakia (carcinoma at diagnosis), while the later flat increase represents those with malignant transformation in the later course of the disease (carcinoma after diagnosis). The overall rate of laryngeal carcinoma diagnosis increased steeply to $11.0 \%$ within 6 months of leukoplakia diagnosis, while it increased by only $7.6 \%$ over the following 4.5 years, which is comparable to the magnitude of the population-based study of Bouquot et al (8). Our data indicated a similar tendency, but with a higher estimated proportion of secondary malignant transformation, which may be explained by the increased mean age of our study participants when compared to the study of Bouquot et al (58 vs. 50 years, respectively). When compared to Isenberg's pooled malignant transformation rate of $8.2 \%$, our estimated rate of $7.6 \%$ was within the same range (7).

The proportion of male patients with vocal cord leukoplakia in the present study was $61.8 \%$, which is at the lower end of the spectrum compared with the results of previous studies $(6,25,26)$. In addition, the present study identified advanced age as a risk factor for the diagnosis of laryngeal carcinoma in patients with vocal cord leukoplakia. This may be easily explained by the latency of progression from precancerous lesions to invasive cancer, and is consistent with the findings of previous studies $(8,27)$.

Our study was subject to several limitations. First, an exact differentiation between carcinoma at diagnosis and carcinoma after diagnosis was not possible, as the data were based solely on ICD codes, whereas clinical information and histological results were not included in the database. In addition, no information on TNM status or stage was available. Moreover, although neoplasms of the supraglottis, subglottis and laryngeal cartilage were excluded, it cannot be ascertained whether the diagnosed cancers have evolved from the previously diagnosed leukoplakia or possibly from another laryngeal site. For the same reason, the diagnostic procedures were not standardized and the accuracy of the diagnoses could not be validated. Finally, information on pre-existing medical conditions and risk factors, including smoking behavior, alcohol use and gastroesophageal reflux, was lacking. Moreover, other relevant parameters, such as voice quality or quality of life, could not be obtained.

An advantage of the study design is the presentation of a large and current sample of routine demographic data from the German healthcare system (real-world data), which allows for a certain reliability of the results and associations. To the best of our knowledge, this is the first time that the association of vocal cord leukoplakia with laryngeal cancer has been analyzed systematically using a representative nationwide practice database. In comparison to previous studies with hospital-based data, a sampling or case selection bias is most likely reduced by the present study design.

In conclusion, this representative nationwide study of otorhinolaryngology practices in Germany revealed that approximately 1 in 5 patients with vocal cord leukoplakia exhibited either carcinoma at diagnosis or malignant transformation within 5 years. A high index of suspicion by physicians is required in older patients, particularly in men. A close follow-up of high-risk patients is recommended, even if the results of the initial biopsy were negative.

\section{Acknowledgements}

Not applicable.

\section{Funding}

No funding was received.

\section{Availability of data and materials}

The datasets used and/or analyzed during the current study are available from the corresponding author on reasonable request.

\section{Authors' contributions}

KK contributed substantially to the conception, design and interpretation of the data and critically revised the manuscript for important content. DUS, AS and MK critically revised the manuscript for important content. LJ contributed to the analysis and interpretation of the data and drafted the manuscript. All authors have read and approved the final version of the manuscript to be published.

\section{Ethics approval and consent to participate}

Not applicable.

\section{Consent for publication}

German law allows the use of anonymous electronic medical records for research purposes under certain conditions. 
According to this legislation, it is not necessary to obtain informed consent from patients or approval from a medical ethics committee for this type of observational study that contains no directly identifiable data. Therefore, no waiver of ethical approval was obtained from an Institutional Review Board or ethics committee. The authors had no access to any identifying information at any moment during the analysis of the data.

\section{Competing interests}

All authors declare that they have no competing interests.

\section{References}

1. Parker NP: Vocal fold leukoplakia: Incidence, management, and prevention. Curr Opin Otolaryngol Head Neck Surg 25: 464-468, 2017.

2. Frangez I, Gale N and Luzar B: The interpretation of leukoplakia in laryngeal pathology. Acta Otolaryngol Suppl 527 (sup527) 142-144, 1997. https://doi.org/10.3109/00016489709124058.

3. Zhang D, Zhou J, Chen B, Zhou L and Tao L: Gastroesophageal reflux and carcinoma of larynx or pharynx: A meta-analysis Acta Otolaryngol 134: 982-989, 2014.

4. Gale N, Michaels L, Luzar B, Poljak M, Zidar N, Fischinger J and Cardesa A: Current review on squamous intraepithelial lesions of the larynx. Histopathology 54: 639-656, 2009.

5. Flint PW, Haughey PH, Robbins KT, Thomas JR, Niparko JK, Lund VJ and Lesperance MM: Cummings Otolaryngology Head and Neck Surgery. 6th edition. Saunders, 2015.

6. Bouquot JE and Gnepp DR: Laryngeal precancer: A review of the literature, commentary, and comparison with oral leukoplakia. Head Neck 13: 488-497, 1991.

7. Isenberg JS, Crozier DL and Dailey SH: Institutional and comprehensive review of laryngeal leukoplakia. Ann Otol Rhinol Laryngol 117: 74-79, 2008.

8. Bouquot JE, Kurland LT and Weiland LH: Laryngeal keratosis and carcinoma in the Rochester, MN, population 1935-1984. Cancer Detect Prev 15: 83-91, 1991.

9. Becher H, Kostev K and Schröder-Bernhardi D: Validity and representativeness of the 'Disease Analyzer' patient database for use in pharmacoepidemiological and pharmacoeconomic studies. Int J Clin Pharmacol Ther 47: 617-626, 2009.

10. Jacob L, Hadji P and Kostev K: Age-related differences in persistence with bisphosphonates in women with metastatic breast cancer. J Bone Oncol 5: 63-66, 2016.

11. Jacob L, Kalder M, Arabin B and Kostev K: Impact of prior breast cancer on mode of delivery and pregnancy-associated disorders: A retrospective analysis of subsequent pregnancy outcomes. J Cancer Res Clin Oncol 143: 1069-1074, 2017.

12. Rathmann W and Kostev K: Association of dipeptidyl peptidase 4 inhibitors with risk of metastases in patients with type 2 diabetes and breast, prostate or digestive system cancer. J Diabetes Complications 31: 687-692, 2017.
13. McGavran MH, Bauer WC and Ogura JH: Isolated laryngeal keratosis: Its relation to carcinoma of the larynx based on a clinicopathologic study of 87 consecutive cases with long-term follow-up. Laryngoscop 70: 932-950, 1960.

14. Gabriel CE and Jones DG: Hyperkeratosis of the larynx. J Laryngol Otol 76: 947-957, 1962.

15. Norris CM and Peale AR: Keratosis of the larynx. J Laryngol Otol 77: 635-647, 1963.

16. Kleinsasser O: Über die verschiedenen Formen der Plattenepithelhyperplasien im Kehlkopf und ihre Beziehungen zum Carcinom. Arch Ohren Nasen Kehlkopfheilkd 174: 290-313, 1959.

17. Kleinsasser O: Über den Krankheitsverlauf bei Epithelhyperplasien der Kehlkopfscheimhaut und die Entstehung von Karzinomen: IV. Mitteilung. Z Laryngol Rhinol Otol 42: 541-558, 1963.

18. Crissman JD and Zarbo RJ: Dysplasia, in situ carcinoma, and progression to invasive squamous cell carcinoma of the upper aerodigestive tract. Am J Surg Pathol 13 (Suppl 1): 5-16, 1989.

19. Resta L, Colucci GA, Troia M, Russo S, Vacca E and Pesce Delfino V: Laryngeal intraepithelial neoplasia (LIN). An analytical morphometric approach. Pathol Res Pract 188: 517-523, 1992.

20. Kambic V and Lenart I: [Our classification of hyperplasia of the laryngeal epithelium from the prognostic point of view]. J Fr Otorhinolaryngol Audiophonol Chir Maxillofac 20: 11451150,1971 . (Our classification of hyperplasia of the laryngeal epithelium from the prognostic point of view).

21. Gale N, Pilch BZ, Sidransky D, Westra WH and Califano J: Epithelial precursor lesions. In: World Health Organization classification of tumour Pathology and genetics of head and neck tumours. Barnes LEJ, Reichart P and Sidransky D (eds). IARC, Lyon, pp140-143, 2005.

22. Fleskens S and Slootweg P: Grading systems in head and neck dysplasia: Their prognostic value, weaknesses and utility. Head Neck Oncol 1: 11, 2009.

23. Gale N, Blagus R, El-Mofty SK, Helliwell T, Prasad ML, Sandison A, Volavšek M, Wenig BM, Zidar N and Cardesa A: Evaluation of a new grading system for laryngeal squamous intraepithelial lesions-a proposed unified classification. Histopathology 65: 456-464, 2014.

24. Blackwell KE, Calcaterra TC and Fu YS: Laryngeal dysplasia: Epidemiology and treatment outcome. Ann Otol Rhinol Laryngol 104: 596-602, 1995.

25. Lampert T, von der Lippe E and Müters S: Verbreitung des Rauchens in der Erwachsenenbevölkerung in Deutschland: Ergebnisse der Studie zur GesundheitErwachsenerin Deutschland (DEGS1). Bundesgesundheitsblatt Gesundheitsforschung Gesundheitsschutz 56: 802-808, 2013.

26. Mourad M, Jetmore T, Jategaonkar AA, Moubayed S, Moshier E and Urken ML: Epidemiological Trends of Head and Neck Cancer in the United States: A SEER Population Study. J Oral Maxillofac Surg 75: 2562-2572, 2017.

27. Gallo A, de Vincentiis M, Della Rocca C, Moi R, Simonelli M, Minni A and Shaha AR: Evolution of precancerous laryngeal lesions: A clinicopathologic study with long-term follow-up on 259 patients. Head Neck 23: 42-47, 2001. 